\title{
Monitoring Cell Development via In Situ Localized Sampling
}

\author{
Mason Chilmonczyk ${ }^{1}$, Gilad Doron ${ }^{1}$, Peter Kottke ${ }^{1}$, Austin Culberson ${ }^{1}$, Kelly Leguineche \\ ${ }^{2}$, Robert Guldberg ${ }^{2}$, Edwin Horwitz ${ }^{3}$, and Andrei Fedorov ${ }^{1}$ \\ ${ }^{1}$ Georgia Institute of Technology \\ ${ }^{2}$ University of Oregon \\ ${ }^{3}$ Emory University
}

June 15,2020

\begin{abstract}
Nascent advanced therapies, including regenerative medicine and cell and gene therapies, rely on the production of cells in bioreactors that are highly heterogeneous in both space and time. Unfortunately, these promising therapies have failed to reach a wide patient population due to unreliable manufacturing processes that result in batch variability and cost prohibitive production. This can be attributed largely to a void in existing process analytical technologies (PATs) capable of characterizing the secreted critical quality attributes (CQAs) biomolecules that correlate with the final product quality. The Dynamic Sampling Platform (DSP) is a PAT for cell bioreactor monitoring that can be coupled to a suite of sensor techniques to provide realtime feedback on spatial and temporal CQA content in situ. In this study, DSP is coupled with electrospray ionization mass spectrometry (ESI-MS) and direct-from-culture sampling to obtain measures of CQA content in bulk media and the cell microenvironment throughout the entire cell culture process ( $\sim 3$ weeks). Post hoc analysis of this real-time data reveals that DSP output is heavily dependent on spatial context. Importantly, these results demonstrate that an effective PAT must incorporate both spatial and temporal resolution to serve as an effective input $f$ or feedback control in advanced therapy production.
\end{abstract}

\section{Introduction}

The treatment of life-threatening ailments is being transformed by advanced therapies including gene therapies for inherited diseases, therapeutic cell treatments (e.g., immunotherapies) for cancers and autoimmune disorders, and tissue engineered medical products to restore, maintain, and replace damaged organs. ${ }^{1-4}$ The outlook for these techniques is promising, with over 900 new investigational drug applications for cell and gene therapy products reported by the FDA as of January 2020. However, even with idealized allogenic models for CAR-T production it is expected that approximately $25 \%$ of the cost to manufacture is associated with quality control alone. ${ }^{5}$ Even if allogenic therapies are shown to be viable alternatives to autologous based therapies, batch variability will result in economic burdens associated with quality control. A shortage in suitable real-time quality control methods capable of monitoring cell bioreactors for feedback control results in large batch-to-batch variability and $a d$ hoc approaches to cell culturing that make scalable and high yield manufacturing difficult and is largely responsible for the high costs. ${ }^{6}$ Since advanced therapy workflows depend on the growth of cells in bioreactors, the process analytical technologies (PAT) for real-time monitoring of cell secreted biomarkers are essential for cost effective biomanufacturing of high quality therapeutic products.

As cell cultures mature and go through developmental stages, the biomolecules they secrete as signaling and paracrine factors serve as the critical quality attributes (CQAs) for cell biochemical state and final therapeutic potency ${ }^{7-9}$ As shown in figure 1 , the concentrations of CQAs vary significantly with both space and time in a cell culture/bioreactor but they are in highest concentration near the cell membrane upon secretion. As the secreted biomolecules diffuse away from the cell surface into the bulk media, they become masked by 
high concentration media constituents that do not indicate cell state such as serum albumin, growth factors, and salts. Furthermore, the microenvironment represents an instantaneous CQA composition while the bulk provides only a temporal average. Widely employed PATs for biomanufacturing, including both real-time and offline variants, do not characterize either the spatial or temporal variations in CQA content, meaning the product processes cannot be effectively monitored.

Most available real-time PATs, such as those that measure temperature, $\mathrm{pH}$, dissolved oxygen, and glucose, are based on simple analytical outputs and lack the specificity and sensitivity required to discover and detect biochemically complex, low concentration CQAs. These outputs are useful indicators of general culture viability but are not useful for predicting the final products' quality. This has motivated considerable efforts to develop other approaches to non-invasive, real-time monitoring including volatile species mass spectrometry, Raman spectroscopy, and infrared or near-infrared spectroscopy. ${ }^{10-12}$ Yet, these approaches are still lacking in their utility for cell bioreactor monitoring, in part due to their poor specificity, limited range of detectable molecules, and low sensitivity for dynamic secretome characterization. A clear and continuing need exists for real-time quality control measurement techniques that are highly sensitive to secreted biomarkers with complex biochemical signatures. An ideal PAT should also be label free or untargeted to enable broad biomolecular detection such that unanticipated or unidentified biomarkers may still be characterized for better process understanding. Electrospray ionization mass spectrometry (ESI-MS) is a particularly promising PAT candidate due to its broad molecular weight coverage, sensitivity, and ability to preserve structure/folding and non-covalent interactions of biomolecular complexes through "soft-ionization". ${ }^{13-15}$ Recently, we demonstrated continuous ESI-MS sensing with a 1 minute response time for detecting the biomolecules serving as proxies to target CQA species. ${ }^{16}$

The Dynamic Sampling Platform (DSP, Fig. 1) is a multi-functional analytical platform for cell bioreactor characterization that can be integrated directly into therapeutic cell manufacturing quality control approaches. The DSP samples very small volumes $(\sim 1 \mu \mathrm{L})$ of liquid from the reactor microenvironment and then processes the sample for real-time analytics. The chemicals that cells produce, secrete, and interact with in their growth environment can be identified using a multitude of analytical tools. As a platform technology, DSP can integrate with the optimal analytical tool for the application. Thus, the capability to monitor spatially-resolved (down to the cellular or sub-cellular level) biochemical activity on the time scale of cellular relevant processes with minimum alteration of the cells' (unobserved) biochemical states, as pursued here, would provide the required level insight for quality control and standardization efforts. In this work, we use DSP in combination with ESI-MS sensing for demonstrating that localized sampling enables in situ fingerprinting of the cell culture over the entire development cycle.

Importantly, using DSP coupled to ESI-MS sensing, we show that CQA heterogeneities exist even within 2D cell cultures. These heterogeneities are important because they impact aspects of cell metabolism, final yield, and product quality. As DSP direct-from-culture sampling does not affect culture sterility or cell growth trajectory during the 3 week culture process, it is found to be suitable for continuous, realtime characterization of CQA content in bioreactors. When incorporated into a workflow with HPLC$\mathrm{MS}^{\mathrm{n}}$ technologies for CQA identification, DSP allows for not only real-time feedback monitoring but also the CQA discovery in biomanufacturing systems. Collectively, these results constitute a vital set of capabilities and biochemical data that i) demonstrate that the local CQA content is critical to detecting cells in their various developmental states (e.g., proliferative, confluent, differentiated) and ii) establish DSP as a viable analytical platform for in situ monitoring of bioreactor state and cell development.

\section{Materials and methods}

\subsection{Dynamic Sampling Platform Design \& Operation}

The Dynamic Sampling Platform (DSP) for ESI-MS incorporates aspects of the previously reported Dynamic Mass Spectrometry Probe (DMSP) to create a system for direct-from-culture monitoring. As shown in Figure 2 , the DSP includes 1) a spatially resolved sampling interface for sterile, direct-from-culture media uptake,

2 ) an optimized "cross flow" sample treatment mass exchanger for inline sample preparation and 3) an inline 
ESI emitter for online MS analysis. The DSP ESI-MS is a remarkably fast, with a temporal resolution of $\sim 1$ minute from sample uptake to MS output. Additionally, an integrated sampling inlet with 10 um resolution enables facile probing of the cell microenvironment in a minimally invasive manner, extracting only $1 \mu \mathrm{L}$ of media per analysis without affecting culture sterility or growth trajectory. This small volume extraction approach ensures that only the local microenvironment is sampled and allows for more frequent sampling without affecting culture viability by removing excessive amounts of media.

A simulation guided approach to the mass exchanger design (see supplementary information) has resulted in a flow arrangement for enhanced sample treatment that significantly improved the device performance prior to the application of DSP to live cell cultures. Successful application of the optimized DSP to murine preosteoblast MC3T3-E1 cell cultures has demonstrated the ability to detect differences between cells in developmental stages relevant to advanced therapy manufacturing, including cells in undifferentiated versus differentiated states as well as indication of cells in their proliferative and confluent states. In order for this approach to yield measurable changes in detection of differentiation biomarkers, localized sampling was necessary to capture the high concentration biomolecules near cell surfaces (Fig. 2, inset).

\subsection{Cell Culture Method}

Murine preosteoblastic cells MC3T3-E1 (ATCC CRL-2593) were obtained and expanded according to established protocols. ${ }^{17},{ }^{18}$ Cells were seeded on T-150 flasks (Corning) at 5000 cells $/ \mathrm{cm}^{2}$ and expanded in a growth media consisting of MEM [?] with nucleosides (Gibco) with 10\% fetal bovine serum (Atlanta Biologicals, Lot \#E15052) and 1\% penicillin/streptomycin (Corning). Media was replenished with clean growth media every 2-3 days until cells reached $80 \%$ confluence. Once confluent, cells were washed with phosphate-buffered saline (PBS, Gibco) and detached from flasks with $0.25 \%$ trypsin-EDTA (Gibco). Dissociated cells were counted and replated onto 6 -well plates at $5 \times 10^{5}$ per well $\left(53,000\right.$ cells $\left./ \mathrm{cm}^{2}\right)$. After overnight adhesion, MC3T3s were separated into two groups, one group subjected to osteogenic differentiation (i.e., differentiated group) using the In VitroOsteogenesis Assay Kit (ECM810, EMD Millipore) and the other cultured in growth media (i.e., undifferentiated group). For cells undergoing osteogenic differentiation, media was aspirated and replaced with $2.5 \mathrm{~mL}$ growth media supplemented with $0.2 \mathrm{mM}$ ascorbic acid 2-phosphate (EMD Millipore), and 10 $\mathrm{mM}$ glycerol 2-phosphate (EMD Millipore) while non-differentiated cells were aspirated and given $2.5 \mathrm{~mL}$ growth media with no additives. Every 3 days, media was replaced after DSP sampling. Immediately after samples of conditioned media from both differentiated and non-differentiated MC3T3s were collected, the media was replaced. After 6 days in culture, the differentiation media was also supplemented with $50 \mathrm{nM}$ melatonin (EMD Millipore). Both cell groups were cultured for 18 days total, being sampled 6 times total. After the final DSP experiment (day 18) cells were washed with PBS and fixed with $4 \%$ paraformaldehyde (Sigma Aldrich) in PBS at room temperature for 15 minutes. Fixative was removed and the cells were carefully rinsed three times with distilled water. Next, differentiated and non-differentiated MC3T3s were incubated in $2 \mathrm{~mL}$ alizarin red staining solution (EMD Millipore) for 20 minutes. Staining solution was then removed and cells were washed 4 times with deionized water. Osteogenic differentiation was confirmed in the differentiated group by red staining of calcium deposition, while no staining was observed in the non-differentiated group, as expected (Fig. S5).

\subsection{DSP ESI-MS Application to 2D Cell Culture}

The MC3T3 cells were cultured in a lab separate from the lab where the mass spectrometer used for online analysis was located, requiring a transport and sterile sampling method that preserved the cell growth processes throughout the entire culturing period. When ready for sampling, the cells were removed from the incubator and placed into a sterile fume hood. The 6 well plate cover was removed and replaced by autoclaved aluminum foil, which was taped down in a manner that allowed for visual inspection through the side of the culture plate. During sampling, a digital microscope was positioned orthogonally to the cell culture plate, allowing for visual confirmation of sampling mode (i.e., local or bulk) as shown in the Figure 2 inset image. After the cells were ready for transport, they were placed in a Styrofoam cooler and brought to the mass spectrometer lab, where they were immediately placed on a hotplate to maintain the media at $\sim 37^{\circ} \mathrm{C}$ throughout the entire sampling process. 
Direct from culture DSP-ESI-MS analysis was carried out every 3 days immediately before media changes, allowing for the highest concentration of biomolecules to be secreted between time points. The MS was tuned and calibrated using Bruker ${ }^{\mathrm{TM}}$ tune mix at the start of each experiment to facilitate accurate mass identification. To remove all air bubbles, which interfere with continuous ESI, the entire fluidic system (shown in Figure 3) was primed with sterilized DI water. To allow access to the cell media through the aluminum foil a 22 gauge sterilized hypodermic needle was used to puncture the foil and the sampling inlet was inserted through the hole. Directly before each sampling event, $2 \mu \mathrm{L}$ of the sterilized water was pumped out of the sampling inlet at $100 \mu \mathrm{L} / \mathrm{hr}$ to purge the sample inlet line, reducing sample carry over effects. Samples of 1 $\mu \mathrm{L}$ volume were drawn into the sampling interface at $50 \mu \mathrm{L} / \mathrm{hr}$ and subsequently infused through the DSP for direct ESI-MS analysis at $30 \mu \mathrm{L} / \mathrm{hr}$. Conditioning flow (1\% acetic acid, $1 \% \mathrm{~m}-\mathrm{NBA}$ ) was run continuously at $50 \mathrm{~mL} / \mathrm{hr}$ throughout the entire experiment. The conditioning flow channel also served as the electrical connection for a picoammeter, which was connected to a stainless steel wire submerged in the conditioning liquid reservoir. The electrospray current during all experiments was maintained at $10-20 \mathrm{nA}$ so that the ESI characteristics remained consistent between experiments. Between each sample, DI water was infused through the DSP and into the MS via ESI to remove residual signal before starting another sampling event. A total of 6 samples were captured from each cell group (i.e., differentiated/undifferentiated) at each time point, 3 local and 3 bulk (Fig. 2, inset), with each sample taken at a spatially disparate location to probe heterogeneities throughout the volume.

\subsection{Mass Spectra Data Processing for Principal Component Analysis}

Bruker MicrOTOF output files were parsed and converted to "mzML" file type ${ }^{19}$ using the open source data conversion software, ProteoWizard.$^{20}$ Principal component analysis (PCA) was completed using two open source software utilities. The first was ms-alone, a python-based utility for preprocessing and peak extraction used on the raw mzML files prior to importing the data to the second utility, multiMS-toolbox , an R based software for PCA analysis. ${ }^{21}$ Preprocessing was accomplished usingms-alone on the raw data including baseline subtraction and peak smoothing. Within the ms-alone setup, the signal to noise ratio threshold of data was set to 0.5 , and a Savitzky-Golay smoothing method was used to reduce noise. ${ }^{22}$ Intensity based (i.e., no data normalization) PCA was run on the entire spectrum (m/z 0-2500) and then winnowed down according to raw loadings plots. The range of $\mathrm{m} / \mathrm{z}$ values with the highest contribution to variance were chosen as the reduced data window size. Without winnowing, the large amount of low intensity noise at the low (m/z 0-500) and high (m/z 1500-2500) ends of the spectra masked the contribution of higher signal to noise ratio features in the middle ranges (Fig. S3).

\section{Results}

A total of 72 spectra were obtained. Representative real-time DSP-ESI-MS data from the first and last time point for both the undifferentiated and differentiated cell groups are shown in Figure S3. The cells in each group were confirmed to be uniformly differentiated or undifferentiated with staining after time point 6 (Fig. S5). Principal component analysis (PCA) was chosen to analyze the spectra because it is a powerful analytical technique capable of detecting subtle differences in the data that contribute the most to variation. ${ }^{22} \mathrm{PCA}$ was implemented to identify differences between the spectra and to identify specific $\mathrm{m} / \mathrm{z}$ values that contribute most to variance between data. These selected $\mathrm{m} / \mathrm{z}$ values were then later used to for comparison with high-performance liquid chromatography (HPLC) data to confirm that spectral features identified via PCA correspond to know CQAs indicating cell state.

Due to the small number of sample replicates at each time point, data from multiple time points were grouped to increase the robustness of PCA. The cells in the differentiated group are expected to have begun differentiation by time point 4 and completed by time point 5 , while the cells in the undifferentiated group remained undifferentiated throughout the experiment. Time points 5 and 6 were therefore grouped to represent the cells in their fully undifferentiated or differentiated state and to omit earlier time points where the cells had not reached their final state. Subsequent staining (Fig. S4) of both the undifferentiated and differentiated cell lines confirmed that the cells had either remained in the undifferentiated state or had completed differentiation after time point 6 , as expected. 
The resulting PCA cluster plots based on bulk samples, taken far from the cells, are shown in Figure 3A. The groupings in these bulk sampling plots are not as well segregated as the groupings for the localized samplings shown in Figure 3B. This suggests that localized sampling is important to being able to detect differences in cell differentiation state. Both cell groups were given different media throughout the culture process, but these differences are not revealed by the bulk samples which suggests that the differences observed in the local samples are due to secreted biomarkers captured near the cells, and not due differences in the cell culture itself. Additionally, the DSP selectively removes smaller molecules from treated samples while retaining larger biomolecules. The additives given to the differentiated cell group have molecular weights below $300 \mathrm{~g} / \mathrm{mol}(\mathrm{Da})$, and were likely removed during DSP treatment. ${ }^{16}$

In order to observe if the same cell culture exhibited differences with time, and to remove the contribution of cell culture conditions to the variance in data, the same differentiated cell culture (i.e., the culture given the osteogenic factors to induce differentiation) was analyzed alone. For this PCA approach, time points 1 and 2 were grouped and compared to time points 5 and 6 . These groupings compare the cells in early time points, when they have not completed differentiation (i.e., undifferentiated cells), to the cells in a fully differentiated state. Since all of the data was taken from the same cell culture in the same continuously performed cell growth and development experiment, this approach also removes the possibility that different culture conditions (e.g., seeding density, media type, etc.) contributed to the differences observed. Figure 4 shows the resulting PCA plots for these groupings. Once again, the spectra do not exhibit significant clustering for bulk sampling (Fig. 4A) but show a stronger clustering for the localized sampling (Fig. 4B). This approach confirms that localized sampling provides an enhanced capability to detect differences between the cells due to secreted biomarkers, which are in highest concentration near the cell membrane.

\subsection{Discussion}

The ability to determine, in real-time, cells' health, therapeutic potential, or developmental state is paramount to developing effective feedback control for advanced therapy (e.g. immunotherapies, biologics, regenerative medicines) production. From a manufacturing standpoint, detecting cells in their proliferative, confluent, or differentiated states is critical to understanding the processes governing their production. For example, clinically relevant cells such as mesenchymal stromal cells (MSCs) have their therapeutic potential drastically altered depending on their differentiation state. ${ }^{23,}{ }^{24}$ As a first step towards real-time monitoring of primary cell cultures, DSP was applied to MC3T3 cells for rapid ESI-MS, followed by PCA of the collected spectra to determine if features in the raw spectra (Fig. S3) corresponded to differences in the secretome, and therefore the cell state. This study was used to carefully study how spatial heterogeneities in a bioreactor affect a PAT output. In this sense, the negative results from bulk samples (Fig. 3A and 4A) are just as important as the positive results from localized sampling (Fig. 3B and 4B). Depending on where a sample is taken in a bioreactor, the resulting PAT output is altered, even if highly sensitive analysis techniques such as ESI-MS are used. This brings to question how even the most sensitive analysis techniques can fully characterize a bioreactor. Therefore, an effective PAT for biomanufacturing, even those that are not real-time, must capture these heterogeneities in the culture environment.

The novel DSP PAT couples high spatial resolution sample uptake with rapid ESI-MS analysis ( $\sim 1 \mathrm{~min})$ by integrating small fluidic tubing ( $360 \mathrm{um}, 50 \mathrm{um}$ ID) with a batch microfabricated mass exchanger that has a total volume of $22 \mathrm{~nL} .{ }^{16}$ The mass exchanger (inset, Fig. 2) operates in a tangential flow orientation with a sample channel separated from a conditioning channel by a nanoporous size selective membrane that fluidically couples the two channels. Active sample treatment enables detection of CQA biomarkers, which involves removal of small molecules such as inorganic salts, retention of larger CQAs, and infusion of ESI-MS enhancing molecules that have been shown to improve sensitivity and limit of detection. ${ }^{16}$ Unlike chromatographic techniques, DSP does not separate biomolecules and therefore suffers from some amount of signal overlap. To overcome this issue, supercharging molecules such as 3-nitrobenzyl alcohol (m-NBA) are infused during sample treatment to help with multi-component detection.

PCA analysis of input spectra from bulk samples, in Figures 3A and 4A, shows no clustering, which indicates that the input spectra are not quantitatively different from each other. However, the cells had undergone 
proliferation to confluence and then differentiated between time points 1 and 6 , and therefore the cells' secretome was expected to change in some fashion during the culture process. The inability of a sensitive technique like ESI-MS followed by careful statistical analysis to reveal any changes in the spectra based on bulk samples suggests that any PAT that uses bulk samples as an input may fail to capture significant aspects of the biochemical makeup. Further, in real-time applications, sampling from the bulk creates a time delay between secretion and detection since the CQA must travel from the cell to the analysis point in order to be detected.

Unlike bulk analysis, PCA using spectra from localized sampling as an input revealed clusters which are associated with cells in their undifferentiated and differentiated states in figures 3B and 4B. An important feature within figures $3 \mathrm{~B}$ and $4 \mathrm{~B}$ is the great deal of variation amongst the same cell group along the horizontal axes (principal component 1 and 2). One explanation for this variation is that every sample taken from the cell culture was taken from a spatially disparate location. The variation observed along the horizontal axes in these cluster plots may reveal subtle changes in the secretory profile that are revealed when sampling at different locations in the well. Of course, there are a number of other factors that could contribute to this variation within the same group, including the fact that the cells in the undifferentiated and differentiated groups experienced different culture conditions, and that the cells were expanding during these experiments.

Separation between cells in their undifferentiated and differentiated states is along the third, orthogonal, principal component 3 (vertical axis). The separation along a single axis suggests that even with variation within subgroups, the dimensionality can be reduced to a single component that captures a significant amount of variation in the input spectra which can be used to predict cells in their undifferentiated or differentiated state. The additives used to induce differentiation in the MC3T3 cells are expected to have been removed due to their small size ( $\mathrm{MW}<300 \mathrm{Da}$ ), and therefore it is unlikely that their presence contributed to the separation observed between the different cultures shown in figure 3B. In order to remove the potential variation due to different culture conditions, the differentiated cell group was analyzed in an early time point vs a late time point to again compare cells in their undifferentiated and differentiated states. In fact, each sample was taken from the same well, so this experimental design removes any changes in cell culture conditions that could contribute to variation in the data. The resulting PCA cluster plot with input spectra from early and late time points from the differentiated cell group (Fig. 4B) separates well along the vertical axis (principal component 3), confirming the assumption that the clustering observed in figure 3B was most likely due to cell differentiation and not culture conditions. Furthermore, this analysis emphasizes a central finding of this work which is that cell bioreactors are highly heterogeneous in nature, and PAT measurements are dependent on where a sample is taken.

Along principal component 1 in figure 3B the undifferentiated cell group (red dots) separates into two subgroups. The two subgroups turned out to be time points 1 and 2 . The cells were seeded such that they were proliferative early in the culture process and later reached confluence. Since this subgroup separation correlated with proliferation, it was expected that similar variation could be observed in the undifferentiated culture alone. The same approach to PCA was done (i.e., time points 1 and 2 versus time points 5 and 6) for the undifferentiated cell group (Fig. S3). In this case, bulk sampling once again resulted in no separation, and for local sampling the separation between the two groups is along principal component 1. This suggests that the variation along principal component 1 may be due to a change in the cell secretome during expansion, which has been shown to correlate with preosteoblasts in a state of proliferation (early) or confluence (late). ${ }^{25}$ In other words, while principal component 3 correlates well with cell differentiation, principal component 1 correlates with cell proliferation. Further studies will be designed to identify targets which are known to correlate with cells in specific states. One drawback of the experimental design used here is the MS used for real-time analysis was not capable of MS/MS (tandem mass spectrometry) for potential chemical IDs. Coupling DSP with a MS system capable of data-dependent-analysis (DDA) for feature identification will significantly increase the potential for CQA identification. However, the PCA output did enable some initial work towards chemical identification. To this end, we made a comparison with offline analysis to determine whether the relatively qualitative PCA approach detects features identified 
from quantitative analysis techniques such as HPLC-MS.

To enhance biochemical interpretation of the PCA analysis, offline HPLC-MS was performed on aliquots of conditioned media which were gathered during media change and frozen at -40 [?]C until the end of the study. HPLC was carried out on media from both cell types at time points $1,2,5$, and 6 to identify candidate differentiation biomarkers and to quantify differences in biomolecules between timepoints. The candidate biomolecules identified with HPLC were then manually compared to $\mathrm{m} / \mathrm{z}$ values with the highest contribution to variance in the PCA data that are hypothesized to correlate with cell state (i.e., differentiated vs undifferentiated). Since the mass spectrometer used for direct-from-culture DSP analysis was not equipped with tandem mass spectrometry, fragmentation patterns could not be matched to the HPLC-MS data. Therefore, the $\mathrm{m} / \mathrm{z}$ values from the PCA loading data were matched to potential chemicals from HPLC-MS based on accurate mass alone, resulting in tentative IDs only. Multiple candidate molecules were identified using this approach to demonstrate that DSP serves to not only generate cell culture "fingerprint" spectra, but to also identify which detected biomolecules correlate with cell state (supplementary information, table S1). For more complete identification of biomolecules, DSP can be used with inline $\mathrm{MS}^{\mathrm{n}}$ for fragmentation data to generate candidate IDs before HPLC is carried out. Further studies will elucidate how PCA loading data correlates with quantitative HPLC data for independent identification of CQA biomarkers using DSP based analysis.

\subsection{Conclusions}

Current advanced therapy manufacturing faces severe limitations in process control because existing PATs are based on bulk measurements that do not represent the true state of the product. The Dynamic Sampling Platform (DSP) ESI-MS is a sensitive analytical platform for probing heterogeneities in a bioreactor. DSP incorporates highly localized sampling direct from culture, inline sample treatment, and real-time analysis that can be adapted for a range of cell cultures (e.g., 3D bioreactors) and sensing techniques such as ESI-MS (and others, e.g. Raman, NMR). For this study, DSP was coupled to a spatially resolved sampling inlet for sampling directly from a 2D cell culture bioreactor. The minimal dead volume DSP system enabled inline preparation of a sample for direct ESI-MS analysis via a modified DMSP (Dynamic Mass Spectrometry Probe). ${ }^{16}$ DSP with real-time, untargeted, MS sensing capability aims to capture and detect low concentration biomolecules secreted by cells. These biomarkers in the microenvironment correlate to cell states relevant for commercial cell production (e.g. differentiation, proliferation, confluence). Capturing and characterizing these CQAs in the microenvironment, in real-time, is critical to enabling feedback control necessary to scaleup and scale out advanced therapeutics.

Application of the DSP enabled in situ analytics to preosteoblast MC3T3 cells revealed that localized sampling was requisite for detection of differences between the cells in an undifferentiated vs a differentiated state. The mass spectrometer in this study was a time-of-flight (TOF) style, which provides fast analysis and accurate mass of biomolecules but cannot fragment these molecules for database matching. Initial work on matching the PCA based $\mathrm{m} / \mathrm{z}$ values to candidate chemical IDs from offline HPLC-MS-MS of the same samples resulted in multiple matches, providing a powerful addition to existing assays for cell culture analysis. Future work aims to incorporate DSP into GMP workflows with inline mass spectrometers capable of tandem mass spectrometry, allowing for real-time identification of detected biomolecules with both accurate mass and fragmentation patterns, facilitating the complete identification of CQAs in real-time. Importantly, in its current design, DSP can be used in conjunction with traditional approaches such as HPLC-MS to bring an important new dimension of dynamic monitoring to clinically relevant workflows. Ultimately, DSP should allow for unambiguous elucidation of how secreted biomolecules correlate with cell state and growth trajectory (e.g., hMSC differentiation or T-Cell CD4 vs CD8 sub-type concentration) and enable the realtime monitoring of these biomarkers for improved manufacturing within all cell-based workflows, including nascent cellular therapies as well as the production of biologics. ${ }^{26-28}$

\section{Acknowledgement}

The work described is supported by NSF Center for Cell Manufacturing Technologies (CMaT) Award 
1648035, Marcus Center for Therapeutic Cell Characterization and Manufacturing Collaboration Grant in Cell Manufacturing, The Georgia Tech Foundation, and the Georgia Research Alliance. Partial support was also provided by Grant Number RO1GM112662 from the National Institute of General Medical Science (NIGMS), a component of the National Institutes of Health (NIH). Its contents are solely the responsibility of the authors and do not necessarily represent the official views of NIGMS or NIH. Device micro-fabrication was performed in part at the Georgia Tech Institute for Electronics and Nanotechnology, a member of the National Nanotechnology Coordinated Infrastructure, which is supported by the National Science Foundation (Grant ECCS-1542174).

\section{Conflict of interest}

Efforts are underway to commercialize the Dynamic Sampling Platform, but the conflict of interest is being managed according to Georgia Institute of Technology's policy.

\section{References}

1. Iyer Nisha, R., S. Wilems Thomas, and E. Sakiyama-Elbert Shelly,Stem cells for spinal cord injury: Strategies to inform differentiation and transplantation. Biotechnology and Bioengineering, 2016. 114 (2): p. $245-259$.

2. Kaiser, A.D., et al., Towards a commercial process for the manufacture of genetically modified $T$ cells for therapy. Cancer Gene Therapy, 2015. 22 : p. 72.

3. Oettgen, P., Cardiac Stem Cell Therapy. Circulation, 2006.114 (4): p. 353.

4. Poulos, J., The limited application of stem cells in medicine: a review. Stem Cell Research \& Therapy, 2018. 9 (1): p. 1.

5. Harrison, R.P., et al., Chimeric antigen receptor-T cell therapy manufacturing: modelling the effect of offshore production on aggregate cost of goods. Cytotherapy, 2019. 21 (2): p. 224-233.

6. Aijaz, A., et al., Biomanufacturing for clinically advanced cell therapies. Nature Biomedical Engineering, 2018. 2 (6): p. 362.

7. Mucida, D., et al., Reciprocal TÉlt;subËgt;HËlt;/subEggt;17 and Regulatory T Cell Differentiation Mediated by Retinoic Acid.Science, 2007. 317 (5835): p. 256.

8. Agarwal, S. and A. Rao, Modulation of Chromatin Structure Regulates Cytokine Gene Expression during T Cell Differentiation.Immunity, 1998. 9 (6): p. 765-775.

9. Albrecht, S., et al., Proteomics in biomanufacturing control: Protein dynamics of CHO-K1 cells and conditioned media during apoptosis and necrosis. Biotechnology and Bioengineering, 2018. 115 (6): p. 1509-1520.

10. Abu-Absi, N.R., et al., Real time monitoring of multiple parameters in mammalian cell culture bioreactors using an in-line Raman spectroscopy probe. Biotechnology and bioengineering, 2011.108 (5): p. 1215-1221.

11. Biechele, P., et al., Sensor systems for bioprocess monitoring. Engineering in Life Sciences, 2015. 15 (5): p. $469-488$.

12. Forcinio, H., Pharmaceutical industry embraces NIR technology. Spectroscopy, 2003. 18 (9).

13. Fenn, J.B., et al., ELECTROSPRAY IONIZATION FOR MASS-SPECTROMETRY OF LARGE BIOMOLECULES. Science, 1989.246 (4926): p. 64-71.

14. Hoofnagle, A.N. and M.H. Wener, The fundamental flaws of immunoassays and potential solutions using tandem mass spectrometry.Journal of immunological methods, 2009. 347 (1-2): p. 3-11.

15. Yates, J.R., C.I. Ruse, and A. Nakorchevsky, Proteomics by mass spectrometry: approaches, advances, and applications. Annual review of biomedical engineering, 2009. 11 : p. 49-79. 
16. Chilmonczyk, M.A., et al., Dynamic mass spectrometry probe for electrospray ionization mass spectrometry monitoring of bioreactors for therapeutic cell manufacturing. Biotechnology and bioengineering, 2019.116 (1): p. 121-131.

17. Wang, D., et al., Isolation and characterization of MC3T3-E1 preosteoblast subclones with distinct in vitro and in vivo differentiation/mineralization potential. J Bone Miner Res, 1999.14 (6): p. 893-903.

18. Gregory, C.A., et al., An Alizarin red-based assay of mineralization by adherent cells in culture: comparison with cetylpyridinium chloride extraction. Anal Biochem, 2004.329 (1): p. 77-84.

19. Martens, L., et al., mzML-a community standard for mass spectrometry data. Molecular \& Cellular Proteomics, 2011.10 (1): p. R110. 000133.

20. Holman, J.D., D.L. Tabb, and P. Mallick, Employing ProteoWizard to convert raw mass spectrometry data. Current protocols in bioinformatics, 2014. 46 (1): p. 13.24. 1-13.24. 9.

21. Hrdlickova Kuckova, S., et al., Evaluation of mass spectrometric data using principal component analysis for determination of the effects of organic lakes on protein binder identification.Journal of Mass Spectrometry, 2015. 50 (11): p. 1270-1278.

22. Cejnar, P., et al., Principal component analysis of normalized full spectrum mass spectrometry data in multiMS-toolbox: An effective tool to identify important factors for classification of different metabolic patterns and bacterial strains. Rapid Communications in Mass Spectrometry, 2018. 32 (11): p. 871-881.

23. Chang, C.-P., et al., Hypoxic preconditioning enhances the therapeutic potential of the secretome from cultured human mesenchymal stem cells in experimental traumatic brain injury. Clinical Science, 2013. 124 (3): p. $165-176$.

24. Paul, G. and S.V. Anisimov, The secretome of mesenchymal stem cells: potential implications for neuroregeneration. Biochimie, 2013.95 (12): p. 2246-2256.

25. Siddhanti, S.R. and L.D. Quarles, Molecular to pharmacologic control of osteoblast proliferation and differentiation. Journal of cellular biochemistry, 1994. 55 (3): p. 310-320.

26. Salgame, P., et al., Differing lymphokine profiles of functional subsets of human CD4 and CD8 T cell clones. Science, 1991.254 (5029): p. 279-282.

27. Rosenbaum, A.J., D.A. Grande, and J.S. Dines, The use of mesenchymal stem cells in tissue engineering: a global assessment.Organogenesis, 2008. 4 (1): p. 23-27.

28. Li, H., et al., Jagged1 protein enhances the differentiation of mesenchymal stem cells into cardiomyocytes. Biochemical and biophysical research communications, 2006. 341 (2): p. 320-325. 


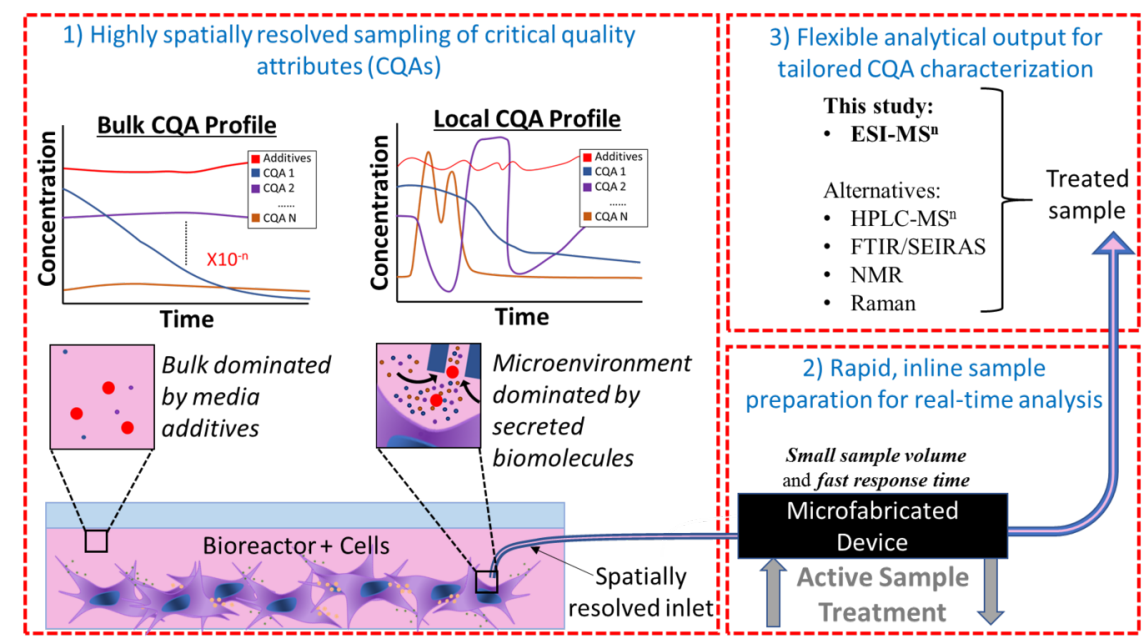

\section{Figure legends}

Figure 1: Motivation for localized sampling and the analytical framework for the Dynamic Sampling Platform (DSP). While cell cultures mature, secreted biomolecules (linked to cell status and growth trajectory) are in highest abundance near the cells' surface. Traditional analysis techniques analyze bulk media, which includes temporally averaged biomolecular content and higher relative concentrations of molecules contained in stock media (e.g., fetal bovine serum (FBS), salts) that can mask the signature of quality indicating analytes. Rapid, small volume, localized sampling is an advantageous approach because it enables spatial and temporal probing of the cell culture, detecting the molecules when and where they are secreted. After sampling, DSP rapidly treats the sample for inline CQA characterization. As a platform technology, DSP is easily integrated with a broad spectrum of analytical tools, but for this study it was coupled with online electrospray ionization mass spectrometry (ESI-MS).
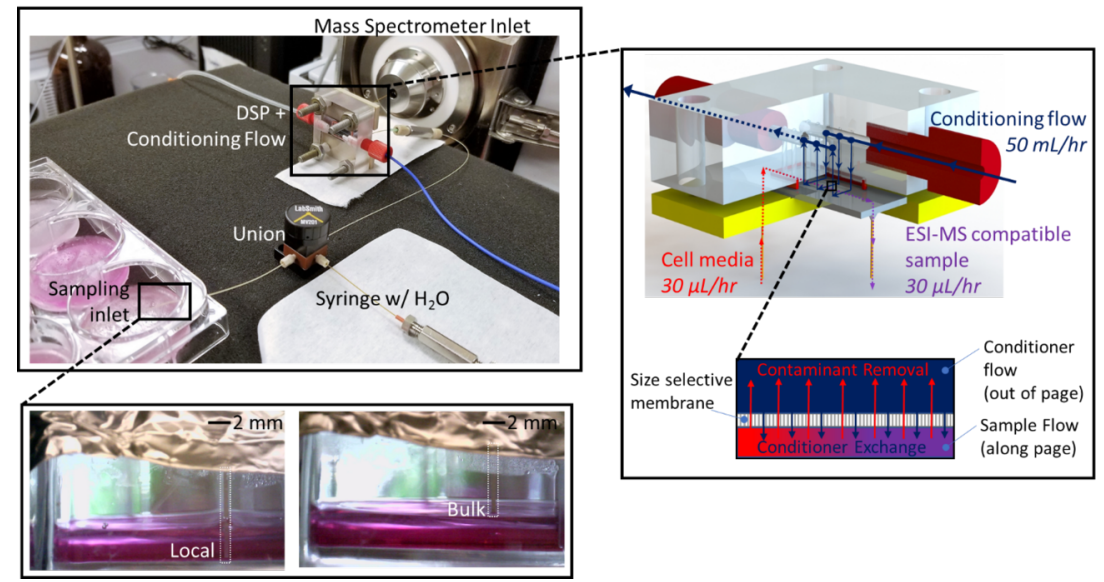

Figure 2: Dynamic Sampling Platform (DSP) ESI-MS setup for direct 2D cell culture analysis. The DSP, is contained in a fluidic package which allows for continuous sample treatment in a tangential flow mass exchanger orientation (right inset image). DSP, in the package, is positioned so that the ESI emitter is directly in front of the MS inlet. The DSP is connected to the sampling interface, which consists of a 50 $\mu \mathrm{L}$ syringe, union, and sampling inlet. Localized sampling is carried out by positioning the inlet directly above the bottom of the cell culture, in close proximity to the cells, while bulk sampling is carried out by 
submerging the capillary just below the media surface (bottom inset image).

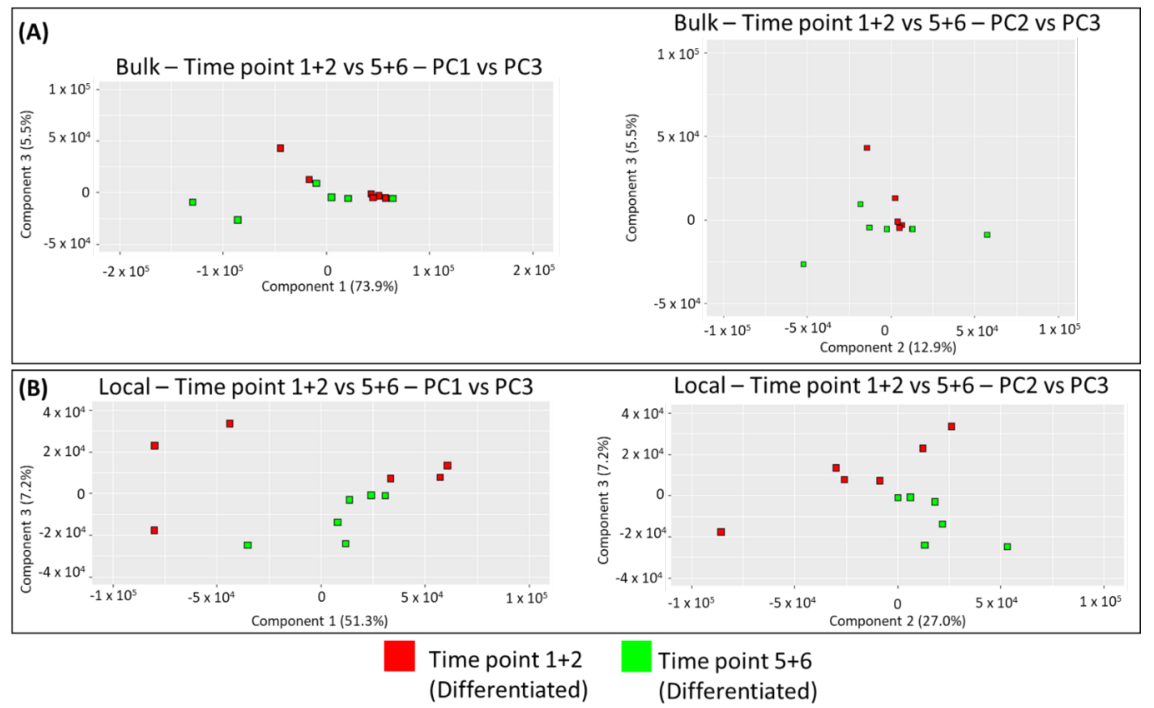

Figure 3 : Principal component analysis (PCA) cluster plots for time points 5 and 6 of the undifferentiated cell group vs time points 5 and 6 of the differentiated cell group. A) Bulk sampling reveals minimal clustering B) Localized sampling reveals clusters for the two groups, indicating that localized sampling is important for detecting differences between the cells at each state.

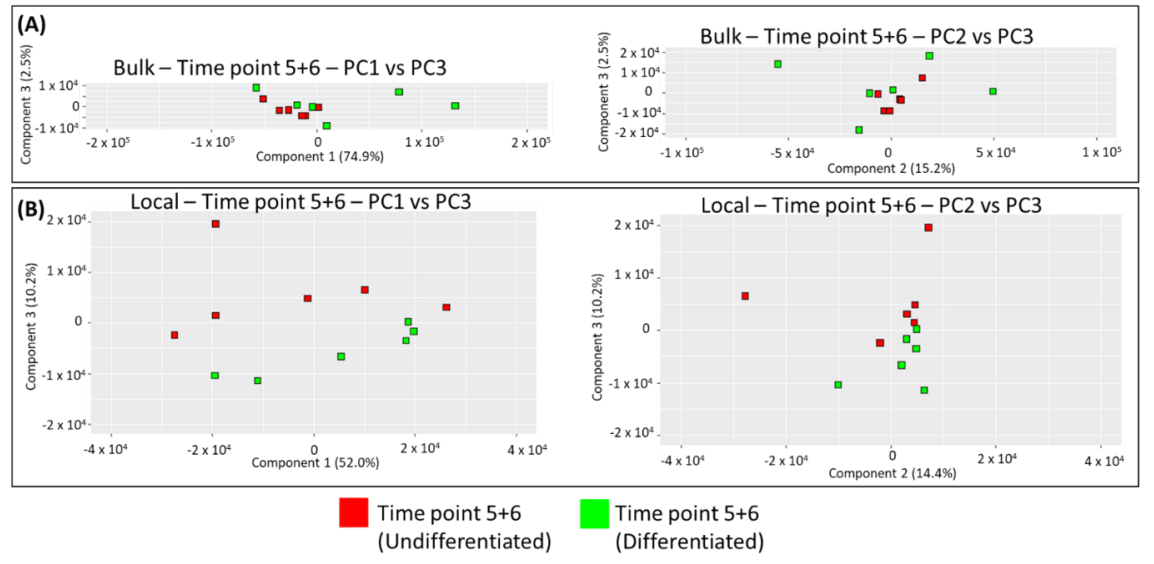

Figure 4 : Principal component analysis (PCA) cluster plots for the differentiated cell line at time points 1 and 2 versus time points 5 and 6 (same analysis done for undifferentiated group in supplementary information). Cells were expected to begin differentiation between time points 3 and 4 , with differentiation completed by time point 5: A) Bulk sampling does not exhibit any clustering, and B) With localized sampling, clusters are observed indicating that DSP detects differences between undifferentiated and differentiated cell lines by probing the near cell enriched secretome. 